\title{
Digital subtraction contrast echocardiography: a new method for the evaluation of regional myocardial perfusion
}

\author{
MARK J MONAGHAN, PETER J QUIGLEY, JENNIE M METCALFE, \\ STEPHEN D THOMAS, DAVID E JEWITT \\ From the Department of Cardiology, King's College Hospital, London
}

SUMMARY The potential of contrast enhanced digital subtraction echocardiography to demonstrate and quantitate myocardial perfusion was evaluated in 36 patients undergoing routine coronary arteriography or angioplasty. In 24 technically successful studies, multiple cross sectional echocardiographic images, obtained before and after intracoronary (sonicated contrast) injection, were stored by high speed, real time data transfer to an on line minicomputer. Subsequent digital subtraction processing of the stored image data provided composite images in which the distribution of myocardial perfusion was easily seen. Quantitative analysis of peak enhanced myocardial grey level and washout half time successfully differentiated between myocardial segments in which angiography had suggested normal, reduced, and grossly impaired or absent perfusion.

The results suggest that this new method of digital image capture and quantitative processing has substantial advantages over previous off line qualitative techniques. It is likely to be of considerable value for routine coronary arteriography, angioplasty, and coronary thrombolysis.

Myocardial contrast echocardiography is now recognised as a convenient and safe method of obtaining information about regional myocardial perfusion. ${ }^{12}$ Contrast enhancement of images of the myocardium is almost certainly due to an acoustic impedance mismatch between the myocardial tissue and the gaseous bubbles generated within the contrast medium. ${ }^{3}$

Over the past few years several improvements in the technique have increased its applicability. The most important of these has been a change from hand agitated to sonicated contrast medium. ${ }^{3}$ Renografin-76, which is widely used for coronary angiography, has been used as an echocontrast medium. Preparation of contrast by hand agitation has an adverse effect upon the myocardium because it produces large bubbles (about $20 \mu \mathrm{m}$ in diameter $)^{45}$ These seem to cause capillary

Requests for reprints to Mr Mark J Monaghan, MSc, Department of Cardiology, King's College Hospital, Denmark Hill, London SE5 9RS.

Accepted for publication 12 August 1987 occlusion. Sonication of the contrast medium creates much smaller and more reproducible bubbles with diameters of approximately $10 \mu \mathrm{m}$. These have capillary transit times similar to red blood cells. ${ }^{6}$ No deleterious effects upon the myocardium have been described with this new method of preparation. ${ }^{2}$

In this study sonicated Omnipaque-350 (iohexol) was used as the contrast agent. This is a non-ionic $x$ ray contrast medium which seems to be more suitable for myocardial echocardiography than Renografin-76. Unlike Renografin it has a limited hyperaemic response and the microbubbles are more stable. These are highly desirable attributes in an ultrasound contrast agent, especially for the measurement of myocardial washout times. ${ }^{7}$

Subjective evaluation of the distribution of myocardial contrast has proved possible in many patients. ${ }^{8}$ The technique has been limited, however, by several technical factors which reduce the quality and usefulness of the contrast images. These include a dependence on the ultrasound equipment instrument settings, image degradation by the output and video recorder circuitry (with inadequate bandwidth), and difficulties caused by high 
background grey levels in the myocardium. Attempts to quantitate perfusion by measuring grey level variables have met with limited success in humans, ${ }^{9}$ presumably for the same reasons.

Use of the method we describe attempts to circumvent many of these problems with a stable sonicated contrast medium and, more importantly, by acquisition of raw digital scan line data from within the scanner. Subsequent subtraction processing of the image data within an on line computer was performed to try to facilitate quantitative measurement of myocardial perfusion and also to produce an image in which the spatial distribution of perfusion is easily seen.

\section{Patients and methods}

\section{PATIENTS}

Thirty six patients undergoing coronary arteriography were recruited. Of these, eight were rejected because technically adequate parasternal echocardiographic images could not be obtained while they were supine. The remaining 28 (10 women and 18 men) patients were aged from 38 to 76 years (mean 56). Four of these patients were undergoing angioplasty procedures-two to the left anterior descending coronary artery, one to the right coronary artery, and one to a left circumflex graft.

A total of 110 intracoronary contrast injections were made in these patients. Thirty nine (35\%) of the image sequences collected from these injections were considered to be inadequate, which resulted in the total exclusion of data from a further four patients. This was either because of inappropriate contrast administration (11 sequences), faulty computer data collection (six sequences) or, most commonly, frame misalignment ( 22 sequences). For successful subtraction processing it is essential that all the images are lined up exactly, with no spatial movement of the endocardial borders between frames. Improvements in the technique of contrast administration and additional computer software to correct for frame misalignment now mean that very few image sequences have to be rejected.

The coronary angiograms in those 24 patients in whom the 71 successful injections were made were analysed by two independent observers. They identified 30 myocardial segments or coronary territories where, unequivocally, one would expect normal perfusion. The coronary arteries supplying those segments were entirely without lesions and, in addition, the segments showed normal wall motion and systolic thickening on the echocardiogram.

A further 36 segments or territories were selected where the coronary supply was abnormal, and reduced perfusion would be expected. In these cases the coronary lesions were proximal and caused between $70 \%$ and $90 \%$ stenosis or $>90 \%$ stenosis with evidence of some limited collateral supply.

Finally, 28 segments were identified in which no perfusion or grossly impaired perfusion would be expected. Again the coronary lesions had to be proximal and causing at least $95 \%$ stenosis. There was no collateral filling and wall motion and thickening were abnormal in these segments on the echocardiogram.

Those echo segments that corresponded to the coronary territories identified above were analysed by subtraction processing of the contrast data.

\section{CONTRAST AGENT}

Coronary angiography or angioplasty was performed from the femoral approach in all cases. Most patients had two injections of sonicated contrast into the left coronary artery and two into the right coronary artery or coronary graft. Data were acquired in two different echo scan planes (as described later). This added a maximum of five minutes to the standard catheterisation procedure.

The contrast medium was $3 \mathrm{ml}$ of sonicated Omnipaque-350 (iohexol) $x$ ray contrast medium. This is a non-ionic medium with a concentration of $350 \mathrm{mg}$ of iodine per millilitre and a viscosity of 10.6 mPa.s at $37^{\circ} \mathrm{C}$. During the contrast injections there were no important changes in either blood pressure or electrocardiographic configuration.

Sonication was performed with a Vibracell sonicator, with a $1 / 2$ " horn and an energy of $250 \mathrm{~W}$ for 20 seconds. The technique and method of microbubble formation by sonication have been described elsewhere. ${ }^{3}$ Samples of sonicated Omnipaque contrast medium were examined by light microscopy and this showed a mean (SD) bubble size of $9.2(3.8 \mu \mathrm{m})$. For comparison, sonicated Renografin-76 was also examined and found to contain bubbles of a similar mean (SD) size $(10.3(4.5) \mu \mathrm{m})$. The concentration of bubbles in Omnipaque seemed to be less than that in the Renografin; however, the bubbles were much more stable with approximately $90 \%$ persisting at three minutes. These differences between the two contrast agents have been confirmed by in vitro experimentation. This demonstrated that Renografin-76 generates higher initial ultrasound backscatter intensity which decays very quickly to $50 \%$ within two minutes. Measurement of myocardial washout time and use of the same prepared sample of contrast agent for multiple injections demand an agent with stable microbubbles. 


\section{ECHOCARDIOGRAPHY}

The echo contrast recording for each coronary artery was performed at the end of the normal sequence of angiographic image planes. In most cases, both mitral valve and papillary muscle parasternal short axis frame sequences were collected for injections into the left and right coronary arteries. During the procedure the transducer was kept stationary on the chest wall and the patient was asked to limit respiration. This reduced movement of the heart relative to the image plane. The echo images were gated to the $R$ wave of the electrocardiogram so that only the end diastolic frames were collected. A total of approximately 27 sequential frames was transmitted to the computer with contrast being injected after the fourth frame (fig 1).

A $3.5 \mathrm{MHz}$ medium focus echo transducer was used in all cases. The ultrasound instrument was a Hewlett-Packard 77020 phased array scanner that was specially modified to incorporate a high speed, bidirectional data link allowing on line, real time transmission of image scan line data to an HP 1000 minicomputer in an adjoining room. The computer was equipped with 2 Mbytes of random access memory, 45 Mbytes of hard disc memory, full floating point processing, and a standard range of output peripherals.

A computer terminal (to the HP 1000) was also positioned in the catheter laboratory and this was used to control the transmission of data between the echo equipment and the computer. With this terminal it was possible to select any portion of the

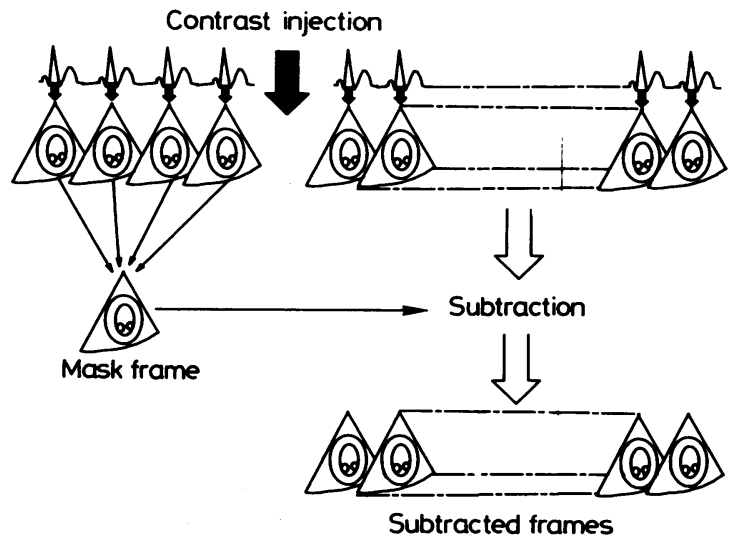

Fig 1 Diagram showing how multiple sequential $R$-wave gated frames are transmitted to the computer. Contrast was injected after the fourth frame. The pre-injection frames are averaged to produce a mask which is subtracted from the post-injection frames. The resulting sequence of subtracted frames shows only those areas where there is enhancement of grey level contrast in the myocardium.

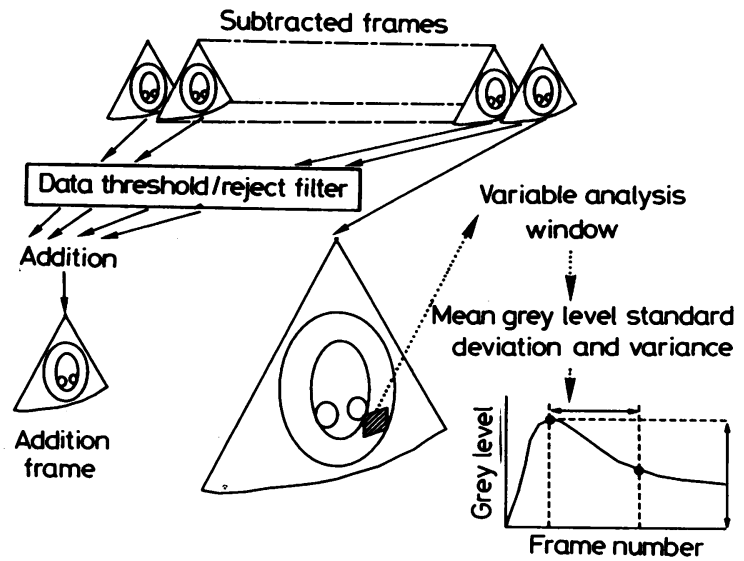

Fig 2 Diagram showing how the subtracted frames are further processed in two ways. A variable analysis window is used to calculate mean grey level, standard deviation, and variance for every frame in multiple positions on the image. The mean grey level is plotted (after smoothing) and the peak level and time (in beats) to half peak value is computed. After subtraction the frames can be added together (after passing through a low level reject filter) to produce a composite addition frame that clearly shows the distribution of perfusion.

images for transmission to the computer. By only storing the relevant part of the images (that is just the short axis of the left ventricle and no right ventricular or extracardiac structures) considerable computer memory was saved. This memory saving allowed us to capture more frames.

\section{DATA PROCESSING AND ANALYSIS}

Figures 1 and 2 show how the captured frames were subsequently processed in the computer system. At any time the entire image sequence of processed or unprocessed frames could be reviewed by replaying through the scan converter in the ultrasound system. The alignment of all the images was checked before processing. Any sequences in which one or more of the frames had moved in relation to the others were discarded.

The four pre-injection frames were averaged to produce a mask frame. This was accomplished by calculating a mean grey level value representing each data point down every scan line for all four frames. The resultant pre-injection frame-averaged mask had a smoothed appearance with an expected reduction in random noise (figs 3 and 4).

Digital subtraction was then performed by subtracting the grey level values in the mask frame data points from the corresponding grey levels in all the post-injection frames. Any resultant negative 


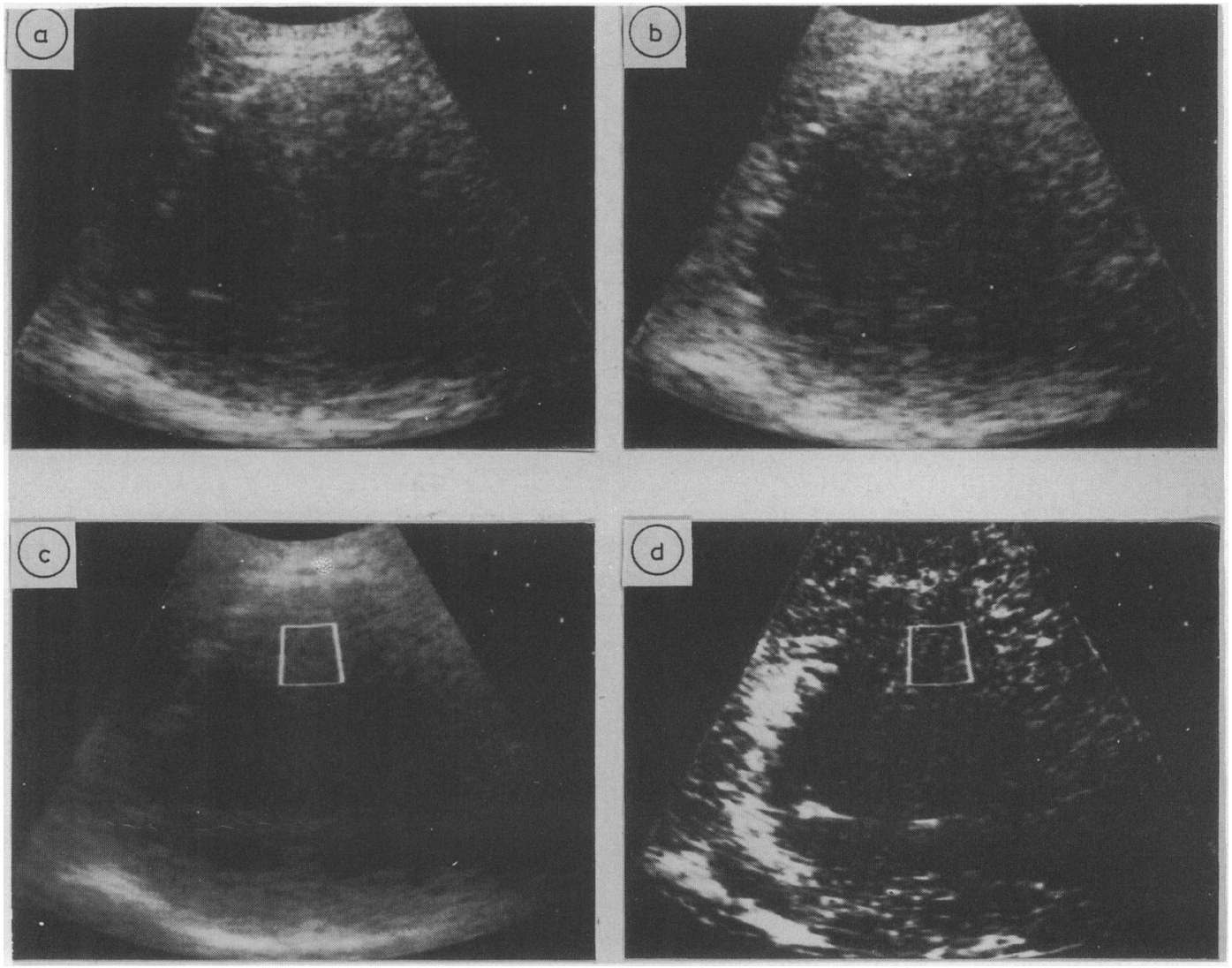

Fig 3 Examples of parasternal short axis images obtained before ( $a$ and $c$ ) and after ( $b$ and d) injection of contrast into a normal right coronary artery. (a) and (b) are digitally captured unprocessed images that have not been degraded by video recording. (a) was obtained before the injection and (b) after injection at peak grey level enhancement. (c) is the pre-injection mask frame and $(d)$ is the composite addition perfusion image which clearly shows perfusion in the territory of the right coronary artery. It is easier to evaluate perfusion from (d) than by assessing the differences between images $(a)$ and $(b)$. The variable analysis window is shown in (c) and $(d)$.

values were set to zero. The resulting series of subtracted frames showed only those areas in which the myocardial grey level was enhanced (by contrast) and this was virtually independent of instrument settings and background myocardial grey level.

To study the spatial distribution of myocardial perfusion, an additional image was created by adding the frames together after subtraction of the mask. Therefore, myocardial segments that were persistently enhanced throughout most of the subtracted sequence became very bright. Conversely, unperfused segments remained dark. To prevent low level noise signals being added together, any grey levels below a threshold value of 15 units were rejected before the frames were summed. This was the lowest threshold that would exclude random noise signals within the left ventricular cavity from the addition. We assumed that the same reject value would also prevent noise in unperfused segments from causing significant brightness when the frames were added together. This addition process produced a composite addition image with a bistable appearanceperfused areas were almost white and unperfused areas were dark (figs 3 and 4).

Previous myocardial contrast studies of experimental preparations have suggested that measurement of the kinetics of contrast enhancement may be used to evaluate perfusion. ${ }^{1011} \mathrm{~A}$ variable window for quantitative analysis that could be changed in size and position was used to facilitate similar measurements in our patients. The mean grey level, 


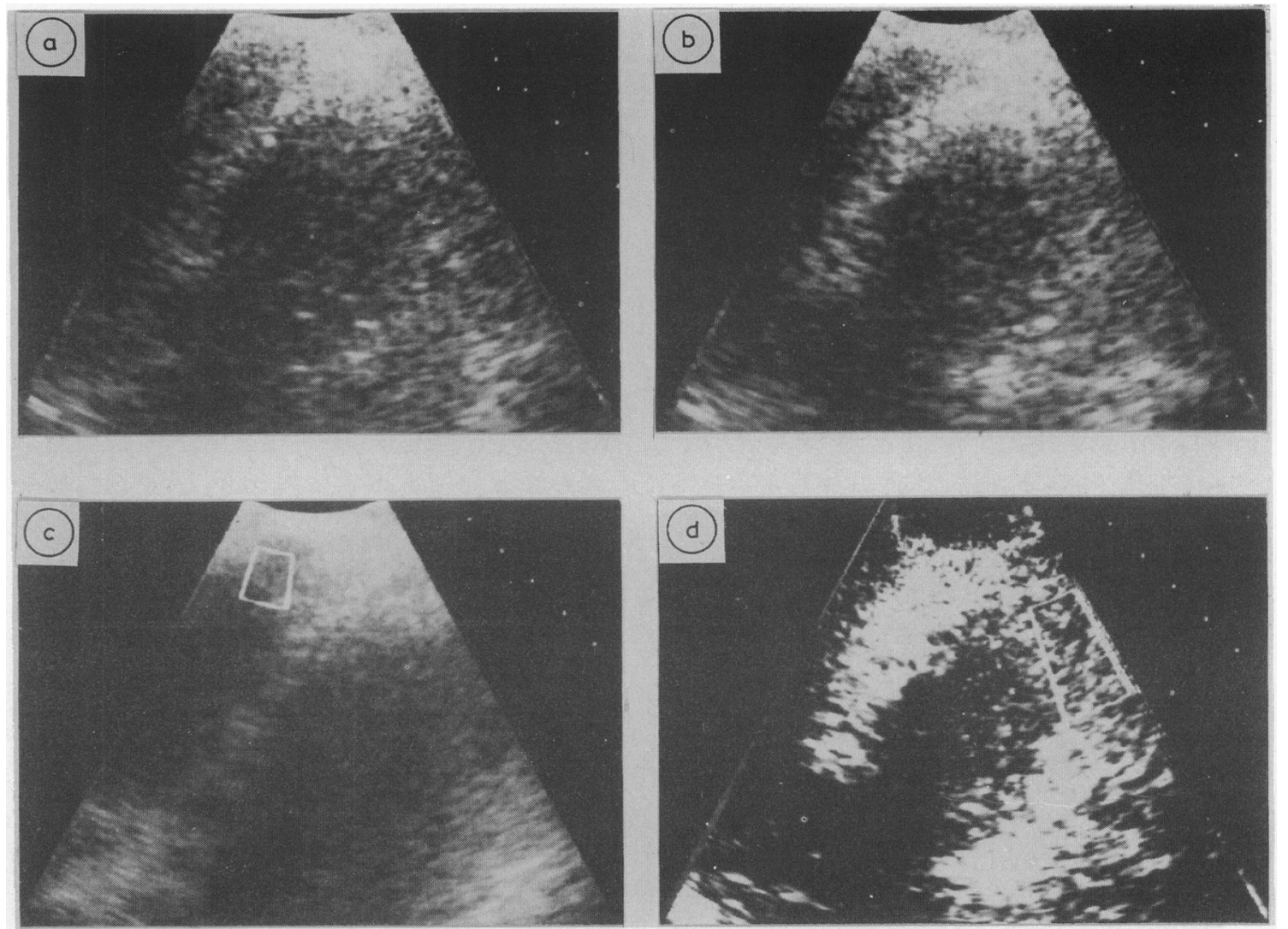

Fig 4 The same sequence of images as shown in fig 3 before and after injection of contrast into the left coronary artery. Subjective determination of contrast enhancement is difficult because there is a low signal to noise ratio in the unprocessed images and the background myocardial grey level is high. The distribution of perfusion, however, is easily seen in the composite addition image (d). The quantitative variable analysis window is shown in (c) and $(d)$.

standard deviation, and variance for the data within the window was calculated by the computer for every frame. The mean grey level was plotted after data had been passed through a three point centre-weighted smoothing algorithm. This feature is shown in fig 2 and examples of the analysis window are seen in figs 3 and 4.

Myocardial regions, broadly identified on standard cross sectional imaging planes, can be correlated with the distribution of the normal epicardial coronary anatomy. ${ }^{1213}$ Figure 5 shows the expected distribution of perfusion zones for each coronary artery for the short axis imaging planes used in this study. To allow for variability in the exact distribution between patients, the quantitative analysis window was positioned in the centre of each region as shown. For measurements in the left anterior descending territory the window was positioned in the most anterior septal segment close to the junction of the anterior right ventricular free wall. Measurements of territory of the left circumflex coronary artery were made between the posterior lateral and medial papillary muscles and an equivalent geometric position was used at mitral valve level. Finally, the window was positioned at the junction of the posterior right ventricular free wall and the interventricular septum to measure perfusion by the right coronary artery.

We measured the peak (subtracted) grey level and the time taken (in terms of number of beats) for the peak to decay to half. This should represent the myocardial washout time of the contrast and be inversely related to myocardial perfusion. These data were calculated for all the segments in which angiography had suggested normal, reduced, and zero or grossly impaired perfusion. 


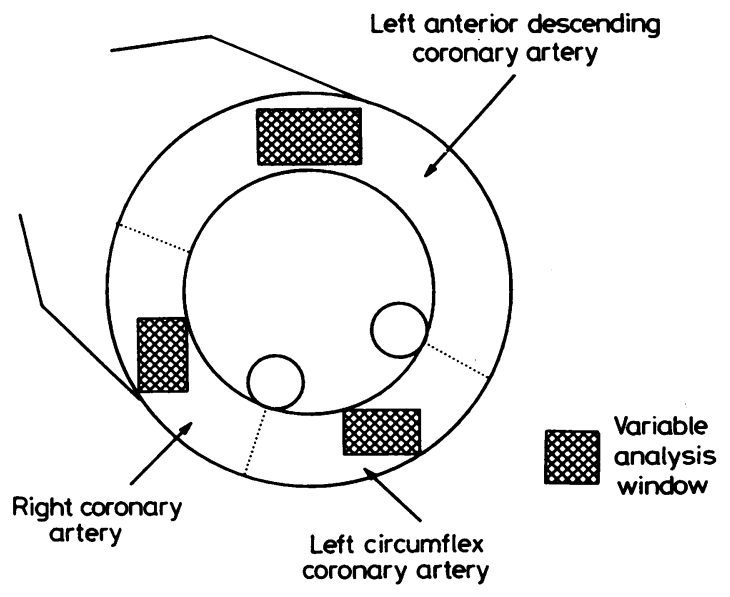

Fig 5 The normal perfusion zones of each coronary artery ${ }^{1213}$ in images obtained in the parasternal short axis plane. The position of the variable analysis window in the centre of each zone is shown.

\section{Results}

Figures 3 and 4 are two typical examples of unprocessed and processed myocardial contrast images. The composite addition image in fig 3 shows normal right coronary perfusion extending high into the medial septum. The spatial distribution of perfusion is much more obvious in the composite image than in the unprocessed examples. Figure 4 shows the identical arrangement of images in a patient undergoing injection of contrast into the left coronary artery. Here the unprocessed images are quite "noisy" and there is a broad range of background baseline echo intensities which makes subjective determination of enhancement difficult. In the composite addition image, however, distribution of perfusion into the territories of left anterior descending and circumflex arteries is clearly seen.

Four of the patients whom we studied were undergoing angioplasty procedures. Figure 6 shows data taken from the anterior septal segment in one of these patients immediately before and after successful dilatation of a lesion of the proximal left anterior descending coronary artery. After the procedure the peak grey level was considerably increased and the washout half time was appreciably shortened, pointing to an increase in segmental myocardial perfusion.

Figure 7 shows the results of quantitative analysis of peak grey level and washout half time in those segments in which angiography showed normal, reduced, or no perfusion. There was a highly significant difference between the three groups in peak grey level, with mean values of 12.54 beats for the normal group, and 8.72 and 4.19 respectively for the groups with reduced perfusion and no perfusion.

The myocardial washout half time for the group of segments with no perfusion or grossly impaired perfusion is theoretically, and also in practice, virtually infinite. It has, therefore, been shown to extend beyond the scale of the graph in fig 7. Again, there was a highly significant difference between the washout time in the groups of segments, with mean values of 8.11 beats for the normal group and $\mathbf{1 5 . 4 8}$ for the group of segments with reduced perfusion.

\section{Discussion}

This study confirmed earlier reports that myocardial contrast echocardiography can be performed with ease and safety. ${ }^{12}$ The methods we describe provide clear images of the perfusion beds of the coronary arteries. The technique also seems to be able to discriminate between different levels of regional myocardial perfusion in humans.

Experimental studies have shown an excellent correlation between the abnormal distribution of contrast in the myocardium and areas of infarction or severe ischaemia. ${ }^{1415}$ However, in humans the images are often technically inadequate especially in patients in whom this technique would be of particular value. If these images with low signal to noise ratio and a broad range of baseline echo intensities are further degraded by video recording, then subjective analysis of contrast enhancement is at best difficult and quantitative analysis is virtually impossible.

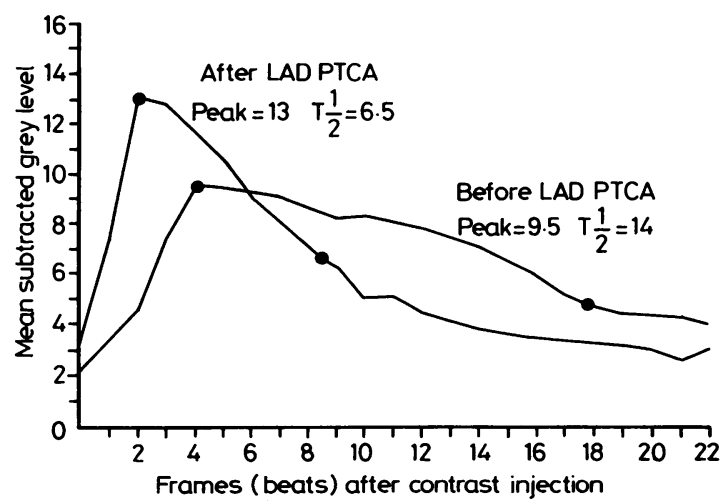

Fig 6 Results of perfusion analysis in the territory of the left anterior descending coronary artery of a patient immediately before and after successful angioplasty of a proximal lesion. The peak grey level and washout half time values are given; they both indicate increased segmental perfusion. 


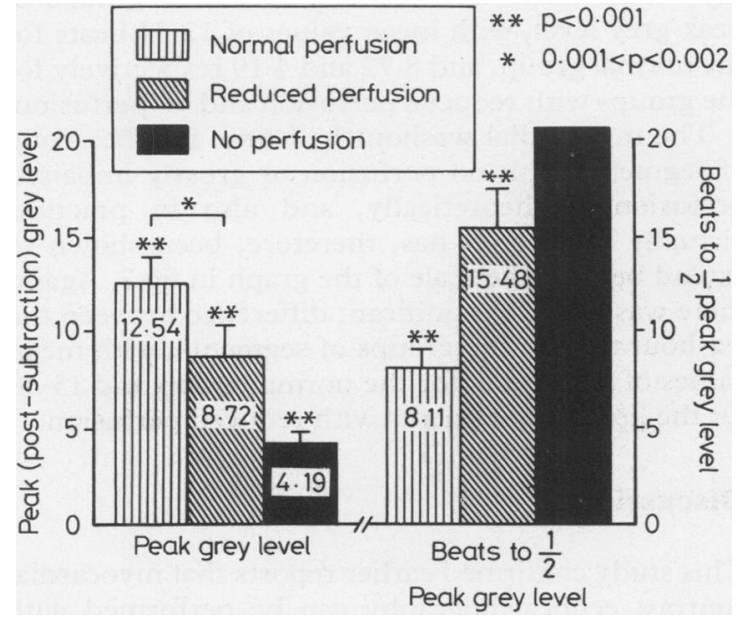

Fig 7 Peak grey level and washout half times (and 95\% confidence interval) for segments of presumed normal, reduced, and zero or grossly impaired perfusion. The differences in these two variables were significantly different for all the comparisons between the three groups. $p$ was $<0.001$ except for differences in peak grey level between the normal and reduced perfusion groups where $p$ was $<0.002$. The washout time for the group with no perfusion is infinite and has been shown extending beyond the scale of the graph.

In the present study on line digital capture of the scan line echo data avoided these problems of image degradation. Subsequent digital subtraction processing seems to reduce the variable effects of instrument settings and facilitates study of the spatial distribution of perfusion, when a subjective approach would be difficult. The ability of digital subtraction angiography to provide high quality contrast enhanced radiographic images is now accepted. The techniques that were applied to echocardiography in this study are completely analogous. In addition, the computer processing of a complete sequence of echo contrast images is rapid enough to allow replay of the processed frames into the catheter laboratory during the same procedure.

The major technical problem with the technique seems to be that of frame misalignment. The creation of additional software that permits spatial translation of individual images has now largely circumvented this problem and together with improved techniques of contrast injection has considerably reduced the number of rejected image sequences.

The results of this study indicate that with digital subtraction, echocardiographic quantification of myocardial perfusion by analysis of the kinetics of myocardial contrast enhancement may be easier than has been previously suggested. ${ }^{911}$ There were, however, several factors that undoubtedly contributed to the highly significant differences in the grey level variables between the groups of myocardial segments in our patients. First of all, the segments were carefully selected, according to the previously described criteria, so that they clearly represented three very different levels of perfusion and any overlap between the segment types was unlikely. Secondly, the method has been improved by (a) the use of a non-hyperaemic contrast agent with greater bubble persistence; $(b)$ direct on line digital capture of echo image data; (c) subtraction processing of the data to enhance differences in myocardial grey levels by removal of background signals; and $(d)$ measurement of washout half time as beats rather than as time units. We feel that this last factor is important because coronary flow is largely proportional to heart rate and would undoubtedly influence contrast washout time. Therefore, there is a strong argument for correcting this measurement for heart rate if it has not been measured in terms of beats.

Further improvements in echo contrast media for human use can be expected soon. These will include purpose-made contrast agents containing microspheres that are very stable and hyperreflective to ultrasound. They can be made of albumin and seem to be capable of traversing the pulmonary capillary bed in sufficient quantities to facilitate opacification of the left heart and myocardium. ${ }^{16}$ The ability to study myocardial contrast distribution after intravenous injections has obvious advantages.

The application of computer technology to the storage and processing of the raw ultrasound data from echo contrast images in a digital format makes the analysis of regional myocardial perfusion in humans a practical proposition. The technique has several advantages over other methods for delineating coronary perfusion beds: it has excellent spatial resolution, it can be repeated at short intervals, and it costs less than radioisotope methods. With the planned development of ultrasound imaging systems incorporating the methods described in this study, the technique will undoubtedly evolve, not only as an adjunct to routine coronary arteriography, but also as a direct means of assessing the effects of intervention.

\section{References}

1 Feinstein SB, Lang RM, Dick CD, et al. Contrast echocardiographic perfusion studies in humans. American Journal of Cardiac Imaging 1986;1:29-37.

2 Moore CA, Smucker ML, Kaul S. Myocardial contrast echocardiography in humans: 1 . Safety-comparison with routine coronary arteriography. J Am Coll Car- 
diol 1986;5:1066-72.

3 Feinstein SB, Ten Cate FJ, Zwehl W, et al. Twodimensional contrast echocardiography. 1. In-vitro development and quantitative analysis of echo contrast agents. J Am Coll Cardiol 1984;3:14-20.

4 Gillam LD, Kaul S, Fallon JT, et al. Functional and pathological effects of multiple echocardiographic contrast injections on the myocardium, brain and kidney. J Am Coll Cardiol 1985;6:687-94.

5 Holt G, Reeves W, Rieder. M, Daley L, Murthy V, Christensen $O$. Negative inotropic effects of intracoronary echo contrast agents [Abstract]. J Am Coll Cardiol 1985;5:474.

6 Feinstein SB, Shah PM, Bing RJ, et al. Microbubble dynamics visualised in the intact capillary circulation. J Am Coll Cardiol 1984;4:595-600.

7 Vandenburg B, Feinstein SB, Kieso R, Hunt M, Kerber RE. Effect of ultrasonic contrast agents, injection rates and coronary vasodilation on myocardial echo amplitude and coronary perfusion area determinations [Abstract]. Circulation 1986;74 (suppl 2):1885.

8 Feinstein SB, Lang RM, Neumann A, et al. Intracoronary contrast echocardiography in humans: perfusion and anatomic correlates [Abstract]. Circulation 1985;72 (suppl III):III-57.

9. Ten Cate FJ, Cornel JH, Serruys PW, Vletter WB, Reiber JC. Quantitative myocardial perfusion imag- ing using contrast 2-dimensional echocardiography [Abstract]. J Am Coll Cardiol 1987;9:112.

10 Ong K, Maurer G, Feinstein SB, et al. Computer methods for myocardial contrast echocardiography. $J$ Am Coll Cardiol 1984;3:1212-8.

11 Ten Cate FJ, Drury JK, Meerbaum S, et al. Myocardial contrast two-dimensional echocardiography: experimental examination at different coronary flow levels. J Am Coll Cardiol 1984;3:1219-26.

12 Feinstein SB, Lang RM, Neumann A, et al. Contrast echocardiography during cardiac catheterisation to assess regional myocardial perfusion in humans [Abstract]. J Am Coll Cardiol 1987;9:113.

13 Goldman ME, Mindich BP. Intraoperative cardioplegic contrast echocardiography for assessing myocardial perfusion during open heart surgery. $\mathrm{J} \mathrm{Am}$ Coll Cardiol 1984;4:1029-34.

14 Schartl M, Fritzsch T, Miszalok V. Quantification of myocardial perfusion by contrast echocardiography. Can J Cardiol 1986;suppl A:25-31.

15 Sakamaki T, Tei C, Meerbaum S, et al. Verification of myocardial contrast two-dimensional echocardiographic assessment of perfusion defects in ischaemic myocardium. J Am Coll Cardiol 1984;3:34-8.

16 Feinstein SB, Keller MW, Dick CD, Bridenstine TR, Wissler RiW:oSuccessful transpulmonary contrast echocardiography in monkeys [Abstract]. J Am Coll Cardiol 1987;9:111. 